FORMATION Formation emploi

Revue française de sciences sociales

104 | octobre-décembre 2008

Pêle-mêle

\title{
Ouvrières à Saint-Nazaire : deux entreprises face à l'intégration des femmes dans les métiers de la navale
}

Female Workers at Saint-Nazaire: how two companies deal with women working in the shipbuilding sector

Arbeiterinnen in Saint-Nazaire : Eingliederung von Frauen im Schiffbau in zwei Unternehmen

Obreras en Saint-Nazaire : dos empresas frente a la integración de las mujeres en los oficios

Philippe Alonzo

\section{OpenEdition}

Journals

Édition électronique

URL : http://journals.openedition.org/formationemploi/1749

DOI : 10.4000/formationemploi.1749

ISSN : 2107-0946

Éditeur

La Documentation française

Édition imprimée

Date de publication : 1 octobre 2008

Pagination : 23-36

ISSN : 0759-6340

Référence électronique

Philippe Alonzo, «Ouvrières à Saint-Nazaire : deux entreprises face à l'intégration des femmes dans les métiers de la navale », Formation emploi [En ligne], 104 | octobre-décembre 2008, mis en ligne le 01 octobre 2010, consulté le 30 octobre 2020. URL : http://journals.openedition.org/formationemploi/ 1749 ; DOl : https://doi.org/10.4000/formationemploi.1749 


\title{
Emplol

\section{Ouvrières à Saint-Nazaire: deux entreprises face à l'intégration des femmes dans les métiers de la navale?}

\author{
Par Philippe Alonzo*
}

La mixité professionnelle n'est pas en soi porteuse d'une plus grande égalité entre les hommes et les femmes. Elle relève d'une politique volontariste des employeurs.

Les femmes représentent aujourd'hui environ $50 \%$ des actifs. Toutefois, le marché du travail féminin reste très cloisonné tant horizontalement que verticalement : $80 \%$ des femmes actives travaillent dans le tertiaire et la moitié d'entre elles exerce un emploi comme salariée d'exécution employée. Entre 1999 et 2003, à Saint-Nazaire, une initiative menée conjointement par l'Agence nationale pour l'emploi (ANPE), les autres acteurs du service public de l'emploi ${ }^{2}$ (SPE) et par les deux plus grands

\footnotetext{
${ }^{1}$ Je remercie vivement Emmanuelle Robert pour son aide précieuse dans la réalisation de cet article.

${ }^{2}$ Les principaux acteurs du service public de l'emploi sont l'ANPE (Agence nationale pour l'emploi et le commerce) et l'Unedic (Union nationale pour l'emploi dans l'industrie). Le SPE comprend également la Direction départementale et régionale du Travail, de l'Emploi et de la Formation professionnelle (DRTEFP), l'AFPA (Association nationale pour la formation professionnelle des adultes), ainsi que des réseaux spécialisés sur certains types de publics, par exemple les missions locales pour les jeunes, l'Association pour l'emploi des cadres (APEC), l'Association pour faciliter l'insertion professionnelle des jeunes diplômés (AFIJ), le réseau Cap-emploi pour les personnes handicapées, etc.
}

employeurs, Les Chantiers de l'Atlantique et Airbus, a tenté de revenir sur les stéréotypes du travail masculin et du travail féminin en proposant des emplois de soudeuses, d'électriciennes ou d'assembleuses à 200 ouvrières industrielles.

L'article s'appuie sur une enquête de terrain, menée en 2003, aux Chantiers de l'Atlantique et auprès d'un de ses sous-traitants ${ }^{3}$ (Voir encadré 1). Il vise à

\footnotetext{
${ }^{3}$ Cette entreprise portera le pseudonyme de Beautuyau.
}

* Philippe Alonzo est sociologue, maître de conférences à l'université de Nantes. Il a publié en 2004 : « Les employés: "un archipel à la dérive" ", in Le retour des classes sociales, Paul Bouffartigue (Dir.), La Dispute, Coll. "États des lieux", pp. 93-109; en 2006, en collaboration avec Olivier Chardon, "Quelle carrière professionnelle pour les salariés non qualifiés? ", Données Sociales, la société française, Insee Références, pp. 265-272. 
explorer les conséquences très différenciées à moyen terme des plans de formation mis en place avec l'aide de l'État dans les entreprises, en termes de mixité. La situation nazairienne est analysée grâce aux modèles de mixité élaborés par les chercheurs du Bureau d'économie théorique et appliquée de l'université Louis Pasteur de Strasbourg ${ }^{4}$. Leur étude identifie trois formes de mixité qui se déclinent à partir d'un ensemble de critères combinant le cadre spatiotemporel dans lequel se déroule l'activité, les tâches définies par la fiche de poste, le travail exécuté ainsi que les statuts d'emploi des salariés. Dans cette modélisation, le niveau minimal est occupé par la mixité de coprésence qui repose sur le fait que les hommes et les femmes partagent uniquement le même espace/temps de travail (bureau, atelier). À un deuxième niveau, la mixité aménagée s'applique lorsque des hommes et des femmes occupent formellement un poste de travail équivalent tout en n'effectuant pas nécessairement les mêmes tâches. Le partage du travail peut être négocié entre les salariés eux-mêmes ou organisé par un supérieur hiérarchique direct. Enfin, la mixité indifférenciée se rencontre là ou les hommes et les femmes effectuent un même travail dans des conditions d'emploi strictement identiques et correspond à la situation la plus égalitaire du partage du travail entre les sexes.

La confrontation entre ces modèles et la situation locale permettra de répondre aux questions suivantes : dans quelles circonstances la féminisation des emplois ouvriers s'est-elle opérée à SaintNazaire? Les employeurs ont-ils utilisé des emplois de femmes comme variable d'ajustement dans une période de pénurie de main-d'œuvre ou ont-ils créé des conditions garantissant leur intégration dans l'entreprise? Ces initiatives ont-elles favorisé l'égalité professionnelle entre les hommes et les femmes ? Enfin, les conditions concrètes de travail et d'emploi des ouvrières, trois ans après leur recrutement, rendent-elles compte de situations intermédiaires entre égalité et inégalités permettant de nuancer les modèles de mixité utilisés?

${ }^{4}$ BETA-Céreq, Mixité et partage du travail, rapport produit pour le ministère du Travail et des Affaires sociales, DARES, I. Études, juin 1997.

\section{Encadré 1 \\ Méthodologie}

Les outils d'investigation mobilisés dans la recherche sont de deux ordres : I'observation directe et l'entretien semi-directif approfondi. Des observations longues (une demi-journée) ont été réalisées lors de plusieurs visites dans les ateliers ou à bord des paquebots. Les entretiens ont été menés avec les ouvrières sur les lieux de travail, sur leur temps personnel. Pour des raisons d'organisation des équipes de travail, les entretiens avec les soudeuses des Chantiers de l'Atlantique ont eu lieu au Bureau d'accueil des personnels extérieurs. Ceux réalisés avec les monteuses en tuyauterie de Beautuyau se sont déroulés dans le réfectoire de l'entreprise.

Les entretiens ont une durée moyenne d'une heure trois quarts. Toutes les ouvrières de Beautuyau (soit 12 personnes) ont accepté de participer à l'enquête et 26 entretiens ont été réalisés avec des soudeuses d'Alstom, dont la moitié a été sélectionnée par l'employeur. Trois entretiens ont été menés auprès de responsables de Beautuyau, un avec le directeur, un deuxième avec un chef d'équipe et le dernier avec le responsable des opérations sur le Queen Mary 2. Par ailleurs, un entretien a été réalisé avec le responsable ressources humaines coque métallique des Chantiers de l'Atlantique.

Enfin, nous avons mené trois entretiens supplémentaires avec des personnes ressources extérieures aux deux entreprises étudiées. Les deux premiers à l'ANPE (Agence nationale pour l'emploil de Saint-Nazaire sur la mise en place de la méthode des habiletés, le dernier à I'AFPA (Association nationale pour la formation professionnelle des adultes) avec un professionnel qui a participé à la mise en place des modules de formation en direction des ouvrières. Au total, 45 entretiens approfondis ont été réalisés pour cette étude. 
PALLIER UNE PÉNURIE DE MAIN-D'OEUVRE MASCULINE

À Saint-Nazaire, l'année 1999 représente une rupture par rapport à la récession des années 90 . Les quatre paquebots en construction, dont le Queen Mary 2, constituent une manne de travail pour l'ensemble des entreprises du bassin d'emploi. Dans la Ville-port, 13000 personnes vivent du secteur de la construction navale. La société Alstom Marine ${ }^{5}$, qui compte 4004 salariés fin novembre 1999, assure uniquement la construction de la coque métallique des navires, soit $40 \%$ de l'activité de montage et d'assemblage des bateaux. Les $60 \%$ restants sont confiés aux entreprises de sous-traitance locales. En 1999, la Direction des ressources humaines des Chantiers et les entreprises de sous-traitance enregistrent une pénurie d'environ 300 emplois qualifiés, principalement des soudeurs, des chaudronniers, des tôliers et des tuyauteurs. Les agences d'intérim ne parviennent pas à combler cette pénurie et, en raison de la reprise économique 6 , l'Agence nationale pour l'emploi

\footnotetext{
${ }^{5}$ Depuis l'été 2006, les Chantiers de l'Atlantique ont été rachetés par la société norvégienne Aker Yards. Cette étude a été menée alors que les Chantiers appartenaient encore à Alstom, ce qui explique la référence à ce groupe tout au long de l'article.

${ }^{6}$ Le taux de chômage du bassin d'emploi de Saint-Nazaire est passé de 17 à $11 \%$ entre décembre 1997 et mars 2001 .
}

(ANPE) de Saint-Nazaire ne dispose pas non plus d'un stock suffisant de demandeurs d'emploi masculins qualifiés pour la navale (Tableau 1).

L'ANPE propose alors à ses partenaires ${ }^{7}$ de recruter des femmes. À Saint-Nazaire comme ailleurs, ces dernières sont davantage touchées par le chômage ${ }^{8}$ en raison de la concentration des emplois féminins. Ainsi, quarante-sept métiers sont demandés à $100 \%$ par des femmes (contre cent huit pour les hommes) et parmi les quarante professions les plus représentatives du bassin d'emploi, six sont occupées à $90 \%$ par des femmes (employé d'étage, secrétaire, agent d'accueil et standardiste, agent administratif d'entreprise, aidesoignante, agent de service de collectivité).

Le SPE propose la mise en place d'un dispositif de formation permettant aux femmes de s'initier aux métiers non traditionnellement féminins. La direction des Chantiers se déclare favorable à la venue de femmes dans les ateliers. Ces dernières en sont quasiment absentes, exception faite de deux ingénieurs femmes qui travaillent dans un des ateliers de soudure. Le plan s'inscrit dans la mise en œuvre
${ }^{7}$ L'ANPE est à l'initiative d'un groupe composé de représentants de l'État, de la région Pays de la Loire, de la Direction départementale du Travail et de l'Emploi (DDTE) et de l'AFPA (Association nationale pour la formation professionnelle des adultes).

${ }^{8}$ En1998, elles représentaient $54 \%$ des demandeurs d'emploi de catégorie 1.

Tableau 1

Ratio entre offres et demandes d'emploi sur quelques métiers du bassin d'emploi de Saint-Nazaire en 1999

\begin{tabular}{|c|c|c|c|}
\hline Métiers & $\begin{array}{c}\text { Offres d'emploi } \\
\text { enregistrées } \\
\text { de janvier à mai 1999 }\end{array}$ & $\begin{array}{c}\text { Nombre de demandeurs } \\
\text { d'emploi (cat. 1 } \\
\text { en avril 1999 }\end{array}$ & Ratio demande/offre \\
\hline Soudeur & 279 & 116 & 0,4 \\
\hline Chaudronnier / tôlier & 244 & 90 & 0,4 \\
\hline Tuyauteur & 134 & 62 & 0,5 \\
\hline Maçon & 189 & 107 & 0,6 \\
\hline Serveur / restauration & 573 & 178 & 0,3 \\
\hline Cuisinier & 385 & 159 & 0,4 \\
\hline Employé polyvalent \\
de restauration
\end{tabular}

Source : service public de l'emploi, Plan d'action local 1999.

$\left(^{*}\right)$ : Les demandeurs d'emploi de catégorie 1 sont les personnes immédiatement disponibles, à la recherche d'un emploi sous contrat à durée indéterminée et à temps plein. 
territoriale des politiques de lutte contre le chômage de longue durée, de prévention et de lutte contre les exclusions et pour l'insertion vers l'emploi des personnes en difficulté (bénéficiaires des minima sociaux, personnes handicapées, jeunes sortis du système scolaire sans qualification) ${ }^{9}$.

Le programme comporte deux modalités d'entrée en formation. La première, à l'initiative du SPE, correspond à des actions de formation mises en œuvre essentiellement par l'AFPA. Soucieux de combattre la précarité de l'emploi, le SPE recommande d'embaucher en contrat à durée indéterminée (CDI) les salariés ayant suivi ces formations. Cette préconisation correspond en outre à l'objectif de réduire les inégalités professionnelles entre les hommes et les femmes. La seconde modalité d'entrée émane d'une entreprise qui souhaite recruter des personnes formées dans tel ou tel métier. L'information a été diffusée par l'ANPE grâce à des campagnes d'affichage dans ses agences, des contacts avec les demandeurs d'emploi, des annonces radio sur les recrutements, des communications dans la presse sur les actions menées, des visites aux Chantiers pour découvrir les métiers de la navale, et des journées découvertes à l'AFPA. La sélection des postulants s'est déroulée dans les locaux de l'ANPE par la méthode des habiletés ${ }^{10}$. Quatre-vingt-seize femmes se sont présentées pour intégrer la formation de monteur tuyauteur proposée par Beautuyau, un peu plus de cent pour devenir soudeuse aux Chantiers. Dans ces deux entreprises, la réussite aux tests n'a constitué qu'un premier palier. Les candidates ont également passé un entretien oral. Chez Beautuyau, le directeur a fixé une tranche d'âge afin de s'assurer que les femmes recrutées soient d'une part en capacité physique d'exécuter le travail demandé et d'autre part entièrement disponibles pour l'entreprise

\footnotetext{
${ }^{9}$ Les ressources mobilisables au niveau de l'État et des principaux partenaires (Association de gestion du fonds pour l'insertion professionnelle des personnes handicapées, région, département, ville) permettent de financer des contrats de qualification, des stages d'insertion et de formation à l'emploi, des programmes d'actions préparatoires et d'initiatives locales, des PRFQ (Programme régional de formation qualifiante) et des aides au recrutement par la formation.

${ }^{10}$ Les candidats ont passé cinq tests, élaborés et étalonnés avec les responsables des Chantiers de l'Atlantique : simulation à la soudure, lecture de plan, mathématiques élémentaires, détection de pièces défectueuses et aptitude au travail en groupe.
}

pendant la durée du contrat : "Quand je les ai recrutées, j'ai essayé d'observer leur situation familiale, voir si ces dames-là n'allaient pas s'arrêter 3 mois après pour nous faire un petit (rires). J'avais interdit le recrutement en dessous de 26 ans et au-dessus de 42 ans. " (PDG, Entreprise Beautuyau) Aux Chantiers de l'Atlantique, un responsable des ressources humaines et un psychologue du travail ont tenté de mesurer l'aptitude des futures ouvrières à intégrer un réseau de sociabilité masculine. Les femmes qui, par exemple, pratiquent ou ont pratiqué le parachutisme, un sport de combat, la moto ; celles qui sont ou ont été pompiers volontaires, etc., ont bénéficié d'une appréciation positive à l'issue de leur entretien.

Entre la fin de l'année 1999 et février 2001, 429 personnes sont entrées dans le dispositif de formation aux métiers de la construction navale, parmi lesquelles 231 femmes (54\%). Selon la Délégation au Développement de la région nazairienne, l'orientation des femmes vers les métiers de l'industrie a concerné environ $20 \%$ des demandeuses d'emploi du bassin. Les femmes sont majoritaires dans presque tous les modules de formation, sauf dans la soudure où l'équilibre est quasi respecté (Tableau 2).

Beautuyau a embauché treize monteuses en tuyauterie. Elles sont les seules ouvrières de cette entreprise, sur un effectif de 250 salariés. En septembre

\section{Tableau 2}

Répartition des personnes entrées dans les formations aux métiers industriels selon le sexe entre 1999 et 2001 (en \%)

\begin{tabular}{|l|c|c|c|}
\hline \multicolumn{1}{|c|}{ Formations } & Hommes & Femmes & Total \\
\hline Soudeurs & 52 & 48 & 100 \\
\hline Monteurs isolation & 34 & 66 & 100 \\
\hline Monteurs tuyauteurs & 30 & 70 & 100 \\
\hline Monteurs de gaines & 49 & 51 & 100 \\
\hline Serruriers bord & 7 & 93 & 100 \\
\hline $\begin{array}{l}\text { Monteurs électriciens } \\
\text { bord }\end{array}$ & 29 & 71 & 100 \\
\hline Charpentiers métaux & 97 & 3 & 100 \\
\hline Total & $\mathbf{4 6}$ & $\mathbf{5 4}$ & $\mathbf{1 0 0}$ \\
\hline
\end{tabular}

Source : AFPA 
2000, Alstom avait déjà recruté trente soudeuses et, au moment de l'enquête, quatre-vingt-dix femmes travaillaient dans les cinq ateliers de soudure. Elles représentaient un peu moins de $20 \%$ de l'ensemble des effectifs féminins de l'entreprise et moins de $10 \%$ des effectifs ouvriers ${ }^{11}$.

La durée des formations est variable selon l'entreprise (12 semaines pour les ouvrières de Beautuyau, 7 mois pour les soudeuses des Chantiers). Elle traduit deux manières distinctes d'intégrer les femmes dans les métiers de la construction navale. La responsable de l'agence locale de l'ANPE de Saint-Nazaire analyse bien le recrutement des femmes sur des postes d'ouvriers. Selon elle, il s'est agi de répondre à un besoin urgent et ponctuel de main-d'œuvre : "On a formé des femmes avec l'emploi en priorité. On avait des offres d'emploi et il y avait un besoin de ces personnes-là en emploi. On a même raccourci des formations pour qu'elles soient adaptables le plus rapidement possible. Entre nous, si les femmes ont eu ce succès, c'est parce qu'il n'y avait pas

${ }^{11}$ En 2006, le bilan social de l'entreprise comptabilise 2881 employés, dont $16 \%$ de femmes. Le nombre de salariés a diminué de $7 \%$ par rapport à l'année 2003. La catégorie des ouvriers $(-8 \%)$ a été la plus touchée par cette baisse. Au total, ce sont 1156 ouvriers $(40 \%), 1171$ administratifs, techniciens, agents de maîtrise (41\%) et 554 cadres (19\%) qui travaillent aux Chantiers de l'Atlantique à cette date. d'hommes pour occuper ces emplois-là. Ce n'était pas un réel choix des employeurs. »

\section{DEUX MANIÈRES D'AMÉNAGER LA MIXITÉ}

Les deux entreprises ont embauché une maind'œuvre locale. La majorité des ouvrières habitent Saint-Nazaire ou dans les alentours, celles qui sont les plus éloignées de leur lieu de travail vivent à une vingtaine de kilomètres.

Les femmes recrutées sont en moyenne plus âgées que leurs homologues masculins (Graphique 1). Elles viennent moins souvent de la formation initiale que les hommes et leurs parcours professionnels alternent des périodes d'activité, d'inactivité et de chômage. Sur trente sept ouvrières interviewées, seules trois déclarent n'avoir connu aucune période de chômage. Elles sont en majorité peu diplômées, titulaires d'un BEPC (brevet d'études du premier cycle du second degré) ou d'un BEP (brevet d'études professionnelles : secrétariat, comptabilité). Auparavant, elles ont exercé des activités de type domestique (garde d'enfants, aide aux personnes âgées, travaux de ménage dans le privé ou dans le secteur public), des

\section{Graphique 1}

Répartition par âge et sexe des personnes entrées dans le dispositif de formation aux métiers industriels

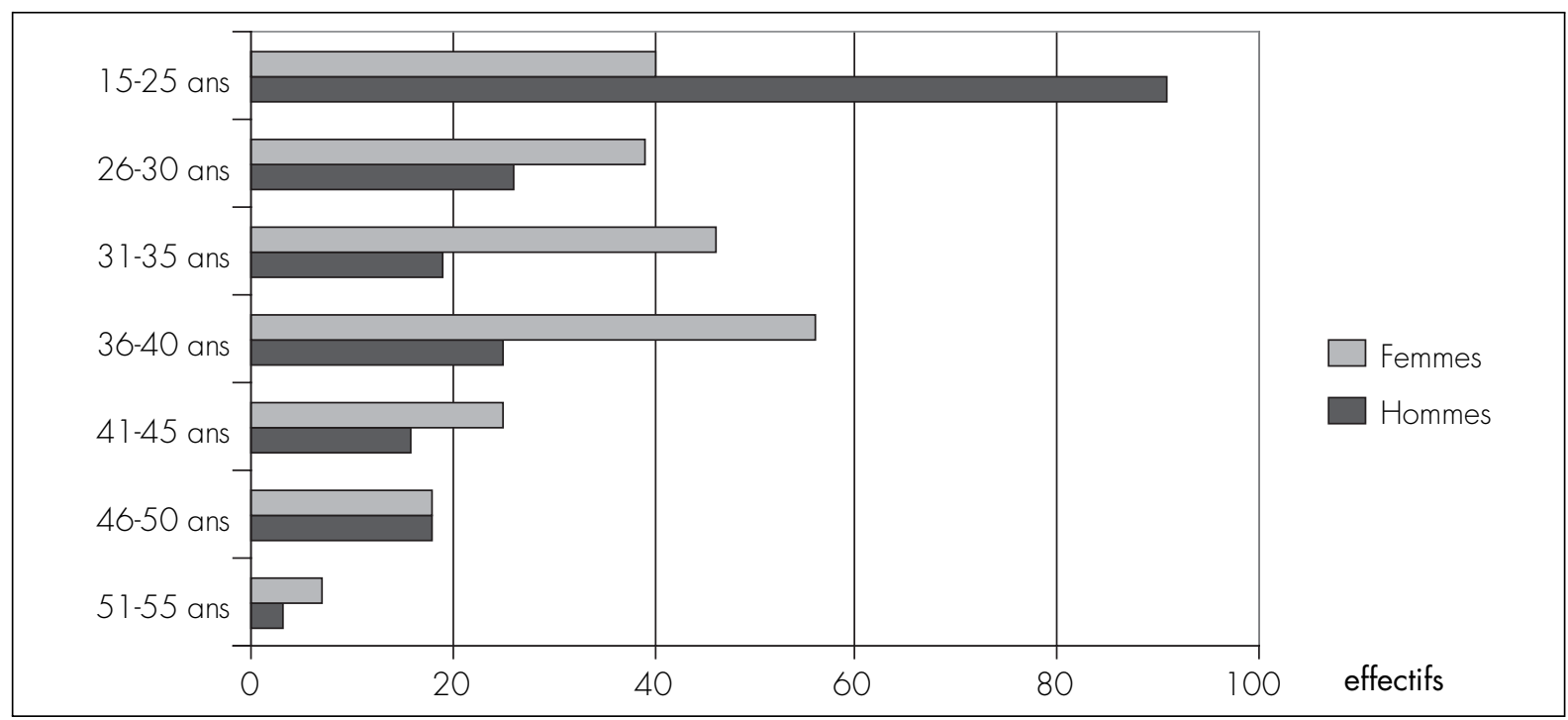

Source : AFPA. 
emplois dans la grande distribution (employé libreservice, caissière), ou dans l'hôtellerie et la restauration (serveuse, femme de chambre). Ces emplois non qualifiés (Alonzo, Chardon, 2006) offrent peu de perspectives professionnelles et cumulent plusieurs désavantages : mauvaises conditions de travail, temps partiel subi, horaires décalés et fragmentés, faibles salaires, missions d'intérim et contrats à durée déterminée. De récentes enquêtes (Béduwé, 2004) montrent que les occasions de sortir d'un emploi non qualifié sont rares et se traduisent par un risque élevé de connaître des périodes de chômage. Ainsi, indépendamment des conditions de travail difficiles dans la construction navale, l'opportunité d'obtenir un CDI à temps complet a constitué le principal attrait pour ces femmes et le principal atout des employeurs. Dans la très grande majorité des cas, elles ont pris leur décision sur un " coup de tête ", sans en référer à leurs proches (conjoint, parents, amis). Ainsi, une ouvrière confie que sa démarche lui a rappelé son premier saut en parachute. $\mathrm{Au}$ moment de leur recrutement, la plupart des ouvrières n'ont aucun repère quant aux conditions de travail dans l'industrie, sauf celles qui, par leur milieu social ou familial, ont un conjoint, un père ou un frère salarié sur le site.

\section{L'entreprise Beautuyau : une mixité de façade}

\section{Une Formation qui ne permet pas de sortir de l'emploi non qualifié}

Les ouvrières ont reçu une formation de dix semaines en salle de cours à l'AFPA et de deux semaines en entreprise. Toutes jugent la formation trop courte. Sylvie estime, par ailleurs, que la non mixité de la formation n'a pas favorisé l'apprentissage du métier. Son contenu théorique est souvent jugé difficile, tandis que la partie pratique du programme est considérée comme étant peu approfondie : «La lecture de plans, c'était un peu prise de tête. On a eu qu'une semaine de soudure et ça n'avait rien à voir avec les conditions dans lesquelles on a travaillé à bord. Durant la formation pratique, on a monté une ligne de tuyaux. Vous imaginez 13 filles sur une ligne! Donc, en pratique on n'a pas fait grand-chose. " (Sylvie, 34 ans, monteuse en tuyauterie $1^{\text {er }}$ échelon) Selon Valérie, l'absence d'hommes, seuls porteurs possibles des «ficelles » du métier, dès leur formation, a posé problème : "Les plans qu'on a eus en formation étaient simples. Ça aurait été bien d'être "mateloté" avec des gars plutôt que de laisser les filles ensemble. Ça nous aurait permis d'apprendre les petits trucs parce qu'on n'a rien vu de concret en formation. » (Valérie, 25 ans, monteuse en tuyauterie $1^{\text {er }}$ échelon)

Pour ces femmes, la formation dispensée a été l'occasion d'acquisitions nouvelles. Toutefois, ces dernières restent limitées au futur exercice d'une activité non qualifiée. Comme le signale la directrice de l'agence locale de l'ANPE : «En trois mois, on n'emmène pas des gens au métier de tuyauteur, qui est très qualifié. Elles ont été recrutées au bas de l'échelle, avec des salaires pas très élevés, sur des emplois pas très intéressants. » Les ouvrières ont le sentiment d'avoir reçu une formation pour des femmes en vue d'exercer un emploi de femmes. Elles considèrent toutes que cette formation reste insuffisante pour pouvoir se considérer comme une vraie professionnelle, capable d'évoluer à moyen ou long terme dans l'entreprise. De fait, aucun diplôme ne vient sanctionner le dispositif, à l'exception de la première licence de soudure à l'électrode $(\mathrm{BV} 3)^{12}$ qui permet aux ouvrières de souder les supports servant au maintien des tuyaux.

\section{Des ouvrières isolées, maintenues dans la précarité}

L'arrivée des femmes survient au moment où l'entreprise utilise un nouveau matériel (le PVC et l'inox) plus léger que l'acier, traditionnellement employé dans la construction navale, et un nouveau procédé de montage : l'emboîtage et le collage ${ }^{13}$. Les termes utilisés par le directeur de l'entreprise pour fonder l'emploi des femmes sur ces postes ne sont pas neutres. Il se dessine un profil de poste en creux, par rapport au travail de tuyauteur considéré comme technique, qualifié et masculin: "Je me suis dit que ce travail ne nécessitait pas une grosse expérience en soudage et les tuyaux sont de faible poids. Donc, pas de main-d'ouvre, plus l'aspect technique, ça peut

12 Le procédé de soudage à l'électrode enrobée, ou baguette de soudage, est le plus simple à mettre en œuvre. Il est constitué d'une âme métallique et d'un enrobage. Il permet de réaliser du soudage de tôles, de tuyauteries et des piquages en toutes positions.

13 A contrario, les tuyaux en acier sont entièrement soudés. 
convenir à des femmes. » Cet employeur reconnaît toutefois des "qualités" aux ouvrières qu'il a recrutées: "Ce travail nécessite du soin. Il faut veiller à ce qu'il n'y ait pas de bavures. Il faut que ça soit propre. Je me suis dit que les femmes avaient plus d'aptitudes pour ce travail-là. En général, les femmes sont plus rigoureuses par nature que les hommes. »

Chez Beautuyau, le recrutement des monteuses en tuyauterie n'a pas changé le rapport sexué aux techniques qui a cours dans l'entreprise. Il consacre même l'exclusion des femmes de l'utilisation des instruments qui fondent la reconnaissance de certains métiers et donc leur exclusion de ces métiers. Cette exclusion est légitimée par des arguments qu'on ne présente plus : capacité physique limitée donc incapacité à travailler l'acier, expérience en soudure limitée, incapacité donc de dominer la nature (Daune-Richard, 1998). Dans cette entreprise, les femmes ne peuvent pas prétendre être de vraies professionnelles. Elles n'ont pas le titre de tuyauteur. Elles sont recrutées à l'échelon le plus bas ${ }^{14}$. La hiérarchie des métiers ne s'en est jamais trouvée bouleversée : les métiers masculins sont qualifiés et les emplois féminins non qualifiés.

Par ailleurs, ces ouvrières n'auront pas la possibilité d'accroître leur niveau de compétence ou leur capacité d'action (Gollac, Volkoff, 2002). Les contrats de travail prévoient qu'une ouvrière "est engagée en qualité de monteuse de petites tuyauteries en PVC et en inox assemblées par collage ou embốtage sur les navires en construction des Chantiers de l'Atlantique ». En libellant les contrats de la sorte, le directeur de cette entreprise s'est protégé contre tous les aléas. Il n'est pas tenu de proposer un autre emploi aux ouvrières dans le cas où une des conditions stipulées dans le contrat viendrait à faire défaut. Il n'a pas non plus obligation de les redéployer sur d'autres activités ou d'autres lieux de production, ce qui reviendrait à les former.

Pour ces femmes, la situation est précaire. Les CDI annoncés sont en réalité des contrats de chantiers. Le droit du travail prévoit que ce type de contrat est uniquement applicable dans les secteurs du BTP et dans la réparation navale. Le contrat de Chantier s'est

${ }^{14}$ Ouvrières professionnelles 1 ${ }^{\text {er }}$ échelon, coefficient 170. développé dans les années 70, avec l'essor des grandes réalisations de l'époque, centrales nucléaires, quartiers d'habitation, etc. Reposant sur des termes imprécis ou équivoques, ces contrats présentent des risques pour les travailleurs dans la mesure où ils permettent à l'employeur de se défaire de ses salariés quand il le décide, sans avoir la contrainte du CDD et de l'intérim qui imposent des indemnités de précarité de $10 \%$. À Saint-Nazaire, le chantier est permanent, même en cas de ralentissement de la production ou de chute des commandes. Aucune des treize ouvrières n'était au courant du particularisme de son contrat de travail au moment de la signature. La grande majorité d'entre elles a cru à un emploi définitif. La désillusion fut à la hauteur des espoirs investis. Lorsqu'elles ont appris la réalité, la plupart d'entre elles se sont senties flouées.

\section{Toutes choses inégales par ailleurs}

Chez Beautuyau, ni la nature du travail des ouvrières, ni les conditions de son exercice et de sa rémunération, ni les conditions d'emploi, ni même les possibilités d'évolution à l'intérieur de l'entreprise ne permettent de parler de mixité. On observe une mixité minimale de coprésence entre les ouvrières, les ouvriers intérimaires monteurs en tuyauterie et les ouvriers statutaires tuyauteurs.

Les relations entre les ouvrières et ces deux groupes masculins laissent cependant entrevoir d'autres types de rapports. Les relations entre les ouvrières et les ouvriers intérimaires sont tendues, donnent lieu à des moqueries voire des insultes de la part des hommes vis-à-vis des femmes. "Les jeunes sont cons, ils ne sont pas intéressants », déclare Nathalie (29 ans,

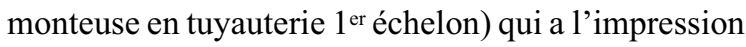
d'être l'attraction d'un zoo: "Ça les fait marrer de nous voir là, ils ricanent bêtement. On se sent observé comme des bêtes sauvages. " Comme le dit Sylvie (34 ans, monteuse en tuyauterie $1^{\text {er }}$ échelon), ces attaques montrent ouvertement que les ouvrières n'ont pas leur place sur le chantier: "On a des réflexions du style: "On prend le boulot des hommes", "Les femmes, elles sont faites pour rester à la maison”. » Selon Sylvie, ces agressions verbales sont la conséquence directe des différences de statut et de salaire entre les intérimaires et les ouvrières : «Je 
pense que ça doit les embêter que les femmes soient rentrées aux Chantiers. Si on fait du BM en 50, ils disent: "Oui mais nous, on le fait en 160". Maintenant qu'on le fait en 160, ils nous disent: "Oui mais nous, on fait de l'acier". Lorsqu'on leur dit qu'ils sont payés plus chers que nous, ils nous disent: "Oui mais nous c'est différent, si on nous demande de faire de l'acier, on peut le faire. Vous, non!" Le problème, c'est que certains ne font pas de l'acier." Les discours de justification qu'utilisent les hommes et la position illégitime dans laquelle ils tiennent les femmes peuvent être interprétés au regard de leur propre situation d'intérimaires. Ils maintiennent les ouvrières à distance par des comportements hostiles quand il s'agit de justifier l'injustifiable : le fait de percevoir un salaire supérieur à celui des femmes pour le même travail. Les intérimaires s'occupent en principe des tuyaux de gros diamètre en acier. Toutefois, les contraintes de la production et le gigantisme du chantier font que les ouvrières vont au-delà du travail qui leur est prescrit. Elles travaillent quotidiennement l'acier, dans les mêmes proportions que les hommes, sans avoir suivi de formation. Elles dépendent alors de la bonne volonté des « anciens » et des recommandations de leur chef direct pour mener à bien leur tâche. De leur côté, les intérimaires assemblent tous les jours des tuyaux en PVC pour honorer les délais de livraison. Si dans les actes, les ouvrières et les intérimaires font le même travail, ces derniers sont payés $1 €$ plus cher de l'heure. Cette différence, injuste aux yeux des ouvrières, constitue une discrimination dont il est difficile de faire la preuve. Elle repose sur le fait que la valeur sociale du travail des intérimaires est définie par les activités les plus qualifiées et par la rareté de la main-d'œuvre qualifiée, contrairement à la valeur sociale du travail des femmes qui reste définie par les activités plancher.

Les ouvrières tentent malgré tout d'imposer leur présence dans un univers qui reste presque exclusivement masculin. Patricia (27 ans, monteuse en tuyau-

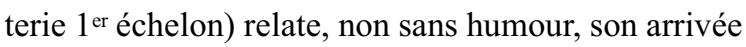
aux Chantiers: "Au départ, il y avait des gars qui nous regardaient du bas de l'échelle. Alors je leur donnais un outil en leur disant: "Puisque tu ne bosses pas, tiens-moi donc ça!"»

A contrario, les relations entre les ouvrières et les ouvriers tuyauteurs de l'entreprise sont plutôt bonnes, empreintes de respect mutuel et de tolérance. Elles donnent lieu à une entraide régulière sur des tâches annexes. Christine (36 ans, monteuse en tuyauterie $1^{\text {er }}$ échelon) se sent même engagée affectivement dans les relations quelle a développées avec les «anciens»: "Les anciens sont vachement gentils. Ils nous disent: "Hé les filles! Si vous avez un problème, vous le dites" Ils nous montrent comment il faut faire. Ils nous charrient gentiment en nous disant: "Vous les femmes, vous n'êtes pas fortes hein!" Mais ils viennent me dévisser la meule ou me porter mon coffre. Les anciens, ils ne disent jamais: "Démerde-toi" Et moi, je ne me sens pas rabaissée de leur demander. » Les rapports entre les ouvriers statutaires de Beautuyau et les ouvrières sont bons parce qu'il n'y a pas d'enjeu entre eux. La séparation stricte des rôles, des statuts et des fonctions permet le développement de relations interpersonnelles placées sous le signe de la bonne volonté. Cette bonne ambiance dissimule en revanche une inégalité de traitement entre les ouvrières et les ouvriers tuyauteurs. Les femmes sont recrutées sur des contrats de travail en trompe l'œil qui ne garantissent pas la continuité de l'emploi, les seconds sur des CDI « normaux ».

L'intégration des femmes dans l'entreprise Beautuyau peut être qualifiée de contingente. Elles ont été utilisées de manière ponctuelle comme variable d'ajustement pour répondre à une pénurie de main-d'œuvre momentanée. Fin octobre 2003, l'employeur envisage des réductions d'effectifs pour faire face à la baisse des commandes de paquebots de croisière. Les ouvrières sont les principales concernées par la réduction de l'activité aux chantiers navals. Ainsi, deux femmes ont été déplacées et travaillent désormais pour Airbus à Saint-Nazaire. Elles occupent des emplois de magasinières non qualifiées pour lesquels elles n'ont reçu aucune formation. Leur situation est précaire et elles n'ont pas pu nous renseigner sur la durée de leur mission. Neuf femmes ont été licenciées. Parmi elles, une suit une formation d'ajusteuse à l'AFPA pour travailler dans l'aéronautique sur l'assemblage de l'Airbus A380. Une femme s'est inscrite dans une agence d'intérim et travaille comme monteuse en tuyauterie pour les Chantiers de l'Atlantique. Sept femmes sont au chômage et envisagent de retourner travailler dans le secteur des services. Une 
femme est toujours ouvrière chez Beautuyau. Elle n'a pas été intégrée dans une équipe mixte. Elle travaille à bord des paquebots sous la responsabilité d'un chef d'équipe. Elle teste les circuits de tuyaux, repère les pannes éventuelles et opère les réparations. Elle a suivi une formation « sur le tas », mais l'évolution de son poste, désormais plus qualifié, ne s'est traduite ni par une augmentation de salaire, ni par un changement de statut. Toutefois, elle considère ce changement comme une chance au regard du licenciement de ses collègues. Enfin, une ouvrière est parvenue à décrocher une formation de tuyauteur pour une durée d'un an. Il s'agit, selon elle, d'une véritable victoire. Elle a négocié son départ en formation auprès du directeur de Beautuyau, à la suite de plusieurs entretiens aux termes desquels elle a réussi à le convaincre qu'une femme pouvait, comme un homme, exercer cette profession. Le ralentissement de l'activité aux chantiers navals n'a pas touché la main-d'œuvre masculine dans les mêmes proportions. Trente ouvriers ont été déplacés vers d'autres ateliers et/ou d'autres villes en attendant une reprise du travail sur le site de SaintNazaire. Aucun n'a été licencié. Les missions des intérimaires n'ont pas été prolongées. Toutefois, la commande d'un porte-hélicoptères et celle d'un méthanier, au début de l'année 2004, ont permis de les réembaucher, contrairement aux femmes.

\section{Aux Chantiers de l'Atlantique : une mixité sous haute surveillance}

\section{Former des ouvrières qualifiées, repérer des futurs potentiels}

Les ouvrières sélectionnées par Alstom (Chantiers de l'Atlantique) ont suivi deux voies de formation. Un contrat de qualification d'un an a été proposé aux moins de 25 ans. Les plus de 25 ans ont été formées aux différentes techniques de soudure à l'Association pour la formation professionnelle industrielle et ont débuté dans les ateliers par un CDD de 7 mois. À la fin de leur formation, toutes ont passé le Certificat de Qualification Professionnelle de la Métallurgie (CQPM) et ont obtenu les trois licences de soudure les plus utilisées dans l'entreprise ${ }^{15}$. La réussite à

${ }^{15}$ La licence 136 : soudure à l'arc électrique. La 114 : au chalumeau. La 131 : soudure au fil fourré avec gaz. l'examen a conditionné leur recrutement sur un CDI à temps complet mais, en raison de la charge de travail, un emploi stable a été proposé aux ouvrières qui n'ont pas réussi le CQPM. Même si celui-ci n'est pas reconnu par l'Éducation nationale comme le CAP (certificat d'aptitudes professionnelles), il l'est par la convention collective ou l'accord de branche auquel il se rattache. Durant la phase pratique de leur formation, les soudeuses tournent dans les cinq ateliers de montage ${ }^{16}$, à raison d'un atelier toutes les six semaines environ.

Les ouvrières sont munies d'un cahier de suivi et sont placées sous la responsabilité d'un tuteur, surnommé "parrain ». Ce dernier est chargé de les accompagner dans la découverte du métier, de les aider en cas de problème et de favoriser leur intégration dans les équipes de travail. À l'issue de la phase de découverte, les soudeuses ont la possibilité de choisir leur futur atelier en exprimant des vœux (jusqu'à trois choix possibles par ordre décroissant). Ces derniers sont soumis à l'appréciation des chefs d'ateliers qui présélectionnent les ouvrières selon leur potentiel d'évolution dans le métier : très bonne technique de soudage, bonne intégration dans des équipes mixtes, forte motivation, bonne autonomie, etc. Le nombre de places étant limité, cette organisation accroît la concurrence entre les ouvrières. Toutefois, le nombre de femmes déçues de n'avoir pu obtenir l'atelier qu'elles souhaitaient reste limité du fait des avantages et inconvénients comparés des divers ateliers et des stratégies individuelles ${ }^{17}$.

16 1) Les Panneaux plans où sont assemblées entre elles les grandes tôles qui formeront les futurs blocs ; 2) la Pré-préfabrication où les panneaux reçoivent les premières pièces, morceaux de cloisons, renforts, longerons ; 3) l'atelier de Pré-fabrication 120 tonnes dans lequel les cloisons se mettent progressivement en place ; 4) l'atelier de Pré-fabrication 180 tonnes dont la vocation est d'assembler les panneaux qui sortent du 120 sous forme de demi blocs et de fabriquer les plus gros blocs du futur navire comme le bulbe avant ; 5) le Pré-montage où les demi blocs sont assemblés entre eux avant d'être hissés à bord du navire en construction. L'ensemble des ateliers fonctionne en $2 \times 8$ heures, sauf celui des panneaux plans où les ouvriers sont soumis au rythme des $3 \times 8$ heures.

${ }^{17}$ L'horaire $2 \times 8$ est très apprécié des ouvrières qui ont des enfants scolarisés, car il leur permet d'être présentes à leur domicile. Le $3 \times 8$ est apprécié par celles qui recherchent un niveau de rémunération plus important. Les ateliers 120 et 180 tonnes correspondent aux choix des professionnelles qui souhaitent exécuter des soudures complexes et diversifiées. A contrario, la « prépré » est réputée pour sa facilité, le revers de la médaille étant que le travail y est assez répétitif. Enfin, le pré-montage a mauvaise réputation car il cumule deux types de difficultés: des techniques de soudure complexes et une exposition directe aux conditions climatiques. 
Les ouvrières n'ont pas toutes débuté au même échelon, alors même qu'elles avaient suivi la même formation. La majorité a été recrutée à l'échelon le plus bas, 170, correspondant au statut de soudeuse qualifiée débutante ${ }^{18}$. Les titulaires d'un baccalauréat ont bénéficié du coefficient 218 : soudeuse qualifiée avec cinq ans d'ancienneté. Selon le responsable des ressources humaines, cette différenciation a permis de repérer, dès leur arrivée, les salariées capables d'évoluer à long terme hors des métiers de la soudure. Cette «avance sur capacités » est un signe positif qui témoigne d'une volonté d'intégrer durablement les salariés, qu'ils soient hommes ou femmes. Toutefois, ce procédé a produit un effet négatif sur les femmes moins diplômées. Ces dernières n'ont pas compris pourquoi la direction valorisait un diplôme sans rapport immédiat avec le travail de la soudure, au détriment de leur formation et de leur CDD : "On nous a plus ou moins emmené en bateau. On nous a dit qu'on serait embauché à 196 ${ }^{19}$. Dans le groupe, une fille a eu 218 parce qu'elle avait le baccalauréat. Je ne vois pas le rapport. Et nous, on a eu 170 ! On est resté baba. On avait toutes au-dessus de 25 ans. En plus on avait un peu d'expérience professionnelle. Même si ce n'était pas dans la métallurgie, on avait travaillé avant.» (Marie-Claude, 33 ans, soudeuse qualifiée débutante coefficient 170)

\section{Aménager une mixité indifférenciée parmi les soudeurs}

Les ouvrières ont intégré des équipes mixtes, comportant autant de soudeurs que de soudeuses, dans lesquelles le travail est le même pour tout le monde. Les tâches sont distribuées par le chef d'équipe en fonction des blocs et panneaux qui attendent dans les nefs. Depuis 2000, Alstom développe l'organisation $\mathrm{du}$ travail en équipes autonomes. Chaque équipe se voit confier une tâche précise et chaque soudeur, homme ou femme, garantit sa prestation par autocontrôle. À l'intérieur des équipes,

\footnotetext{
${ }^{18}$ Ce coefficient garantit un salaire net mensuel de 920 euros. Des primes viennent s'y greffer, variables selon les ateliers et les horaires. Le niveau moyen de rémunération net mensuel atteint 1200 euros.

${ }^{19}$ Le coefficient 196 correspond au statut d'ouvrier qualifié soudeur ayant trois ans d'ancienneté.
}

des ouvriers et des ouvrières peuvent devenir des « correspondants », moyennant une formation supplémentaire. Ils prennent alors en charge des tâches d'approvisionnement, de contrôle de la qualité, de la sécurité, de rangement du poste de travail, ou bien encore de l'outillage. Ainsi une soudeuse correspondante sécurité n'hésite pas à interpeller les hommes, anciens ou non, gradés ou non, qui ne portent pas les EPI (équipement de protection individuel) réglementaires.

La mise en place des équipes autonomes augmente la marge de liberté des ouvrières qui ont le sentiment de pouvoir s'organiser elles-mêmes ou entre collègues. Un planning mensuel fixe la production. L'autocontrôle les soustrait à la surveillance quotidienne hiérarchique. Les équipes fonctionnent sur le modèle $\mathrm{du}$ « rôle tournant » qui prévoit que chaque ouvrier peut développer d'autres compétences et ainsi être polyvalent. À l'atelier des panneaux plans, une ouvrière a passé le permis pont pour apprendre à déplacer les grandes tôles qui doivent être soudées. Une autre a obtenu une formation complémentaire en soudure (la licence 121) afin de travailler, à l'aide de robots à souder (SUMI), selon la technique de l'arc submergé.

Conformément à ce que la direction a annoncé, les ateliers semblent bien fonctionner selon une mixité indifférenciée. L'organisation des équipes montre que la substitution de l'un(e) par l'autre, sans que cela n'entraîne l'exclusion de l'un(e) par l'autre, est effective. Elle est même encouragée par les chefs d'équipe et les responsables d'ateliers qui testent la bonne intégration des femmes dans l'entreprise.

Les responsables ignorent, malgré tout, les rapports interpersonnels à l'intérieur même des équipes de travail. On observe des cas de mixité aménagée entre les soudeurs et les soudeuses. Ces derniers se partagent alors le travail selon un modèle traditionnel : les hommes réalisent les tâches les plus physiques et les plus techniques, les femmes s'accommodent de cette répartition. Les ouvrières légitiment ces différences par la tradition ou le désir de maintenir une bonne ambiance de travail: "Ils ont toujours fait cette tâche, c'est normal qu'ils continuent. » (Patricia, 28 ans, soudeuse qualifiée débutante coefficient 170) «Il maîtrise mieux la SUMI que moi.» (Valérie, 
36 ans, soudeuse qualifiée débutante coefficient 170) "Il aime bien se servir du pont pour déplacer les tôles. Ça ne me gêne pas qu'il le fasse. Ça lui fait plaisir. » (Christine, 43 ans, soudeuse qualifiée débutante coefficient 170). «On ne va pas arriver et dire: "Je veux faire ça, je sais le faire, pousse-toi!"” (Fabienne, 40 ans, soudeuse qualifiée débutante coefficient 170)

Cette répartition informelle du travail est avant tout fonctionnelle et n'empêche pas les femmes de percevoir leur prime annuelle ${ }^{20}$, ou d'obtenir des formations complémentaires. Toutefois, la mise en place d'équipes de travail mixtes ne remet pas en cause la norme dominante des ateliers, qui reste masculine. Toutes les soudeuses interviewées ont le sentiment d'exercer un "métier d'homme ». Pour intégrer cet univers masculin et faire disparaître l'idée que les femmes sont des intruses dans les ateliers, il leur a fallu user d'un subtil équilibre, qu'une ouvrière résume sous la formule : "Faire ses preuves, tout en restant à sa place. » (Emmanuelle, 31 ans, soudeuse qualifiée coefficient 218)

En tant que femmes et débutantes, certaines ont subi le regard scrutateur des hommes - leur jugement sur le travail accompli, leurs conseils même s'ils n'étaient pas sollicités - pendant qu'elles réalisaient des opérations de soudure délicate. Elles ont vécu une véritable mise à l'épreuve visant à tester leur résistance physique et morale ainsi que leurs compétences professionnelles: "Il a fallu que je leur montre que j'étais capable, c'était pas acquis hein! » (Véronique, 26 ans, soudeuse qualifiée débutante, coefficient 170) D'autres ouvrières ont dû supporter des blagues de mauvais goût pendant les pauses. Marie-Claude (33 ans, soudeuse qualifiée débutante, coefficient 170) raconte qu'un jour, elle a envoyé un de ses collègues «se faire voir ailleurs » car elle ne supportait plus de l'entendre lui demander un café et d'être ainsi rabaissée au rôle de «boniche».

De l'avis des ouvrières, il règne aujourd'hui un bon état d'esprit au sein des équipes. Comme le dit Véronique en souriant: "Les équipes sont soudées. Le

${ }^{20}$ La prime ou «galon » est calculée par le chef d'atelier. Son montant maximal est fixé à $45 €$ par an. fait de travailler en équipe autonome nous responsabilise. On sait exactement ce qu'on a à faire et on n'attend pas que quelqu'un vienne nous dire ce qu'il faut faire. C'est valorisant. " L'organisation du travail favorise également la solidarité. Ainsi, les membres d'une équipe partagent un coffre à outils.

La référence masculine est également portée par le responsable des ressources humaines qui avoue rencontrer plus de problèmes avec les femmes qu'avec les hommes. Ces dernières sont plus sensibles aux risques liés aux conditions de travail difficiles. Les campagnes de sécurité, affichées dans les vestiaires et sur les murs des ateliers, accroissent leur conscience des risques et les incitent à considérer ces nuisances comme des «problèmes». Toutes dénoncent les postures pénibles, les fumées nocives, les risques de brûlure, la saleté, le port de charges lourdes, les nuisances sonores, ainsi que les conséquences du travail en plein air. Contrairement aux ouvriers qui fonctionnent davantage dans l'écart à la règle, les soudeuses respectent les normes de sécurité à la lettre et savent, le cas échéant, rappeler le règlement. Ainsi, elles n'hésitent pas à demander des équipements neufs lorsque les leurs sont usés ou endommagés, ce qui leur vaut d'être mises à l'index par certains de leurs collègues masculins. "Au début, dès qu'on allait réclamer une nouvelle paire de gants ou des coussins pour les genoux, les gars se fichaient de nous. Ils nous traitaient d'emmerdeuses. » (MarieClaude, 33 ans, soudeuse qualifiée débutante, coefficient 170) La présence des soudeuses a deux conséquences sur les comportements des soudeurs. Elle permet à ces derniers de réaffirmer, individuellement et collectivement, leur domination et leur image d'ouvriers virils, capables d'affronter les pénibilités du travail, fragilisée par l'arrivée de femmes exécutant le même travail qu'eux (Gollac, Volkoff, 2002). Les revendications des ouvrières concernant le respect des normes de sécurité viennent alimenter le sentiment «qu'ils ne sont pas des gonzesses». La présence des femmes dans les ateliers vient en même temps re-questionner le rapport au corps entretenu par les hommes dans les métiers «physiques». Elle autorise les ouvriers les plus prisonniers de la norme masculine à exprimer leur souffrance, même si cela se passe à l'écart du groupe des hommes, comme le confie Marie-Claude: "Il n'empêche que la crème 
pour les mains en cas de brûlure, ils sont bien contents qu'on en ait. Il y en a même qui nous demandent si on ne peut pas leur ramener un tube quand on va chercher nos fournitures. Mais ils ne veulent pas que les copains le sachent!»

\section{Une intégration durable sous surveillance}

Malgré une volonté de banaliser la présence des femmes dans les ateliers, le responsable ressources humaines a un a priori. Il soupçonne certaines ouvrières de tout mettre en auvre pour tenter de rejoindre un emploi de bureau. Pourtant, parmi les vingt-six soudeuses interviewées, aucune n'avait un tel projet. Deux soudeuses ont quitté définitivement le métier, tout en restant salariées d'Alstom. L'une, enceinte, ne pouvait plus travailler dans les ateliers où les fumées de soudures, malgré l'oxygène ou le port du casque, restent nocives. Elle est aujourd'hui chauffeur de navette et transporte les ouvriers des parkings à leurs lieux de travail. L'autre a fait valoir son diplôme bac +2 et sa connaissance de la soudure au plomb, pour obtenir un poste de contrôleur technique de sous-traitant.

À Alstom, l'intégration des ouvrières semble réussie, mais elle reste cantonnée au métier de soudeur. Comme pour tout ouvrier, les soudeuses se voient imposer un parcours minimal de cinq ans dans le métier. Aux dires du Chef d'atelier du « 120 tonnes », c'est le temps qu'il faut pour faire un bon soudeur. En revanche, elles peuvent acquérir des formations complémentaires et toute la carrière de soudeur s'ouvre devant elles. Certaines femmes ont souhaité suivre une formation en anglais pour pouvoir négocier les panneaux et les blocs directement avec l'armateur avant de les envoyer à l'atelier de prémontage. Cette formation constituait pour elles un moyen d'enrichir leur travail, de le mener de $\mathrm{A}$ à $\mathrm{Z}$. D'autres ouvrières ont fait des demandes de formation en informatique, «en prévision », comme elles disent. Toutes ces demandes ont été refusées par la hiérarchie qui les a jugées trop éloignées de l'exercice du métier proprement dit.

Selon la DRH, l'entreprise a fait son maximum pour intégrer durablement les femmes dans l'entreprise et leur présence dans les ateliers semble acquise. Mais il faudra attendre quelques années avant de pouvoir la qualifier d'innovation. Ces ouvrières auront-elles de réelles possibilités d'exercer des emplois de la maîtrise, de se tourner vers d'autres métiers industriels ? Il sera alors intéressant de confronter leurs trajectoires professionnelles avec celles des ouvriers masculins pour déterminer si les bornes mises en place aujourd'hui par la DRH se sont révélées être des verrous, propices à réintroduire des inégalités professionnelles entre hommes et femmes.

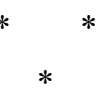

Quel bilan tirer de ces deux initiatives nazairiennes qui ont permis à des femmes d'investir des professions exercées majoritairement par des hommes?

Aux Chantiers de l'Atlantique (Alstom), le mouvement vers la mixité relève d'une politique volontariste de l'employeur. Le parcours formation/emploi sous contrat de qualification jeune et adulte, proposé aux ouvrières, leur a permis d'acquérir une qualification réelle, (presque) équivalente à celles des ouvriers masculins. Par ailleurs, ces femmes se sont insérées durablement dans l'emploi par la signature de contrats à durée indéterminée. Ici, la DRH a fait en sorte que les soudeuses ne soient pas perçues comme des exceptions mais comme une « normalité » dans un univers de travail exclusivement masculin.

Chez Beautuyau, le développement de nouvelles technologies a renforcé les stéréotypes de sexe, alors que chez Alstom, la pénurie de main-d'œuvre a montré que le déterminisme technologique empêchant les femmes d'exercer un métier dit « masculin» n'existe pas en soi.

Chez Beautuyau, la formation élémentaire des femmes a été légitimée par le fait qu'il n'y a pas grand-chose à apprendre. Les tâches qui leur ont été confiées intègrent l'idée d'une limite de leur savoirfaire et entérinent la faible valeur sociale de leur travail. Cette logique ne permet pas de sortir du cercle vicieux des différences entre les métiers masculins, considérés comme "qualifiés », et les emplois féminins jugés non qualifiés. Dans cette entreprise, les ouvrières furent envoyées là où il n'y avait pas d'hommes. Il n'y eu ni action volontariste en matière 
d'égalité professionnelle, ni même volonté d'intégrer les femmes dans l'entreprise.

En trois ans, les ouvrières de Beautuyau sont passées du statut de femmes providentielles, permettant de pourvoir à la pénurie de main-d'œuvre masculine, à celui de femmes surnuméraires. Finalement, cette entreprise les a utilisées uniquement comme une armée de réserve.

Le cas des ouvrières de Beautuyau interroge aussi sur la manière dont l'ANPE et le service public de l'emploi suivent les dispositifs de formation qu'ils mettent en œuvre en partenariat avec les entreprises. Développer des actions permettant de pallier à la fois le taux de chômage élevé chez les femmes peu ou pas qualifiées et le déficit de main-d'œuvre aux chantiers navals, comme s'il s'agissait de «deux vases communicants » (Lemarchant, 2007), sans contrôler (à minima) la manière dont ces femmes ont été intégrées dans les entreprises, relève selon nous de la gageure. Ainsi, il ne suffit pas de rapprocher l'offre de la demande uniquement du point de vue quantitatif et de ne considérer que le nombre d'emplois créés à court terme.

Cette étude sur la mixité dans les métiers de la navale est également l'occasion de réinterroger les rapports sociaux de sexe au travail. La mixité professionnelle n'est pas en soi porteuse d'une plus grande égalité entre les hommes et les femmes. L'exemple des monteuses en tuyauterie démontre parfaitement qu'il ne suffit pas que les hommes et les femmes partagent le même espace/temps de travail pour faire disparaître les discriminations. La mixité, tout comme la parité, ne vient pas régler la question de l'égalité. Au contraire, elle l'ouvre, pour reprendre les propos de Sabine Fortino (2000).

La majorité des femmes interrogées dans le cadre de cette étude - les soudeuses comme les monteuses en tuyauterie - et les réactions masculines auxquelles elles se sont heurtées dans les ateliers, surtout dans les premiers temps, soulignent la persistance des stéréotypes de sexe concernant les rôles traditionnels «féminins » et «masculins ». Comme l'explique
Anne-Marie Daune-Richard, "Le sexe de celui qui occupe tel ou tel emploi constitue un marqueur durable de la représentation de l'emploi» (DauneRichard, 1998). Le cas de Beautuyau montre que cette représentation reste prégnante, même quand les conditions changent ou sont différentes. Dans cette entreprise, le maintien des positions masculines et féminines traditionnelles relève de l'employeur. Les hommes, surtout les intérimaires, ont vécu l'arrivée des femmes dans les ateliers comme une transgression et la plupart des femmes se sont senties comme des intruses. Chez Alstom, la mixité mise en place par la DRH tend à bouleverser les rapports sociaux entre les sexes. Cette confrontation des genres masculin et féminin constitue une expérience sociale importante tant pour les hommes que pour les femmes. Ces dernières ont pu devenir soudeuses, même si elles le doivent à une pénurie de maind'œuvre masculine. Cela traduit une évolution des rapports de force entre les sexes en faveur de l'arrivée des femmes. Cela montre également que l'exclusion des femmes de ces métiers tend à être de moins en moins socialement légitime. Certes, le fait que des hommes et des femmes partagent la même activité dans un même espace de travail ne supprime pas les distinctions, parfois subtiles, dans la distribution des tâches. Dans les ateliers de soudure, des formes de mixité aménagée perdurent et complexifient les relations entre les ouvriers et les ouvrières. Ces dernières subissent encore la domination masculine, mais elles sont également capables de l'utiliser ou d'en jouer pour mieux assurer leur intégration, pour obtenir de l'aide dans la réalisation de leur travail, ou pour questionner les pratiques de travail masculines. L'expérience d'Alstom est encore trop récente pour affirmer que ces différences se solderont par la re-création d'espaces ségrégués marqués par des inégalités entre les soudeuses et les soudeurs. En revanche, la manière dont les ouvrières se sont finalement intégrées tend à montrer que les femmes ont toute leur place dans les ateliers et que ce qui est considéré comme «naturellement» masculin (et « naturellement » féminin) résulte d'une construction sociale. 
Bibliographie

Alonzo P., Chardon O. (2006), "Quelle carrière professionnelle pour les salariés non qualifiés?», Données Sociales, la société française, Insee Références, pp. 265-272.

Béduwé C. (2004), «L'emploi non qualifié dans les trajectoires professionnelles de jeunes débutants ", in Méda D., Vennat F. (dirs.), Le travail non qualifié, permanences et paradoxes, La Découverte, collection « Recherches », pp. 269-284, Paris.

Bureau d'Économie Théorique et Appliquée de l'université Louis Pasteur de Strasbourg-Céreq (1997), Mixité et partage du travail, Rapport produit pour le ministère du Travail et des Affaires sociales, DARES, « Études », juin.
Daune-Richard A.-M. (1998), «Femmes et travail, l'enjeu des qualifications », Revue Pour, Femmes en milieu rural, $\mathrm{n}^{\circ} 158$-juin, GREP.

Fortino S. (2000), La mixité au travail, La Dispute, collection « Le genre du monde », Paris.

Gollac M., Volkoff S. (2001), Les conditions de travail, La Découverte, collection « Repères ».

Gollac M., Volkoff S. (2002), « La mise au travail des stéréotypes de genre », in Ouvrières, les dessous de l'embellie, revue Travail, Genre et Sociétés, l'Harmattan, pp. 25-53.

Lemarchant C. (2007), «La mixité inachevée », in Formation et orientation : l'empreinte du genre, revue Travail, Genre et Sociétés, Armand Colin, pp. 47-64.

\section{Résumé}

\section{Ouvrières à saint-Nazaire :}

\section{deux entreprises face à l'intégration des femmes dans les métiers de la navale}

$$
\text { Philippe Alonzo }
$$

Les initiatives permettant aux femmes d'exercer des métiers " traditionnellement » masculins sont peu nombreuses. À Saint-Nazaire, entre 1999 et 2003, les plans de formation mis en place dans les entreprises, avec l'aide de l'État, ont eu des conséquences diverses pour les ouvrières qui travaillent dans le secteur de la construction navale. Le mouvement vers la mixité et au-delà vers l'égalité professionnelle entre les hommes et les femmes relève d'une politique volontariste des employeurs comme l'illustre, à partir d'une pénurie de main-d'œuvre masculine, la comparaison entre une entreprise de sous-traitance et les Chantiers de l'Atlantique. En l'absence de cette politique, on assiste au renforcement des stéréotypes de sexe et à l'utilisation de l'emploi des femmes comme variable d'ajustement.

Mots clés

Division sexuelle du travail, métier de la construction navale, besoin en main-d'œuvre, formation professionnelle en entreprise

Journal of Economic Literature: J 16, M 53, M 51 